\title{
Conjugation of siRNA with Comb-Type PEG Enhances Serum Stability and Gene Silencing Efficiency ${ }^{\mathrm{a}}$
}

\author{
Karthikeyan Gunasekaran, Thi H. Nguyen, Heather D. Maynard, \\ Thomas P. Davis, Volga Bulmus*
}

A thiol-modified siRNA targeting the enhanced green fluorescence protein (eGFP) gene was conjugated with RAFT-synthesized, pyridyl disulfide-functional poly(PEG methyl ether acrylate)s ( $p(P E G A) s)$. siRNA-p(PEGA) conjugates demonstrated significantly enhanced in vitro serum stability and nuclease resistance compared to the unmodified and thiol-modified siRNA. The complexes of siRNA-p(PEGA) conjugates with a fusogenic peptide, KALA $((+) /(-)=2)$ inhibited the protein expression approximately 28 -fold more than the KALA complex of the unmodified siRNA. The protein inhibition caused by siRNA-p(PEGA)-KALA complexes $(56 \pm 5 \%-58 \pm 3 \%$ of the fluorescence expressed in non-treated cells) was comparable to the effect of the unmodified siRNA-lipofectamine com-

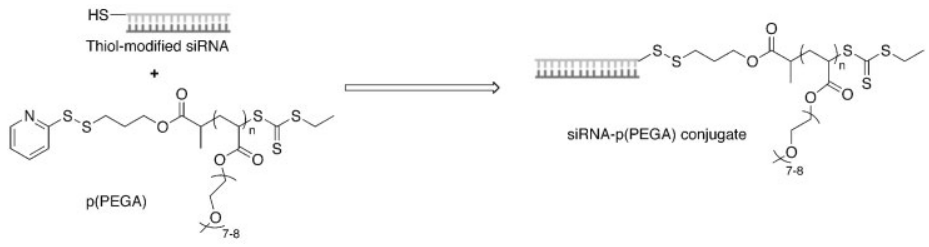
plex $(77 \pm 7 \%)$.

K. Gunasekaran, V. Bulmus

School of Biotechnology and Biomolecular Sciences (BABS), The University of New South Wales, Sydney, NSW 2052, Australia

Fax: +90 232750 6645;

E-mail: volgabulmus@iyte.edu.tr

T. H. Nguyen, H. D. Maynard

Department of Chemistry \& Biochemistry, The California

NanoSystems Institute, University of California, Los Angeles,

California 90095, USA

T. P. Davis

The Australian Centre for Nanomedicine, School of Chemical Sciences and Engineering, The University of New South Wales, Sydney, NSW 2052, Australia

V. Bulmus

Department of Chemical Engineering, Izmir Institute of

Technology, Gulbahce, Urla, Izmir 35430, Turkey

\footnotetext{
${ }^{a}$ S Supporting information for this article is available at Wiley Online Library or from the author.
}

\section{Introduction}

Short interfering RNA (siRNA) is an effective mediator of RNA interference ${ }^{[1]}$ and holds great promise as a therapeutic agent. ${ }^{[1,2]}$ However, the therapeutic potency of siRNA is severely limited by delivery problems. ${ }^{[2-5]}$ PEGylation, a technique commonly used to improve the in vivo pharmacokinetics of protein therapeutics, ${ }^{[6,7]}$ has also been investigated for siRNA delivery. ${ }^{\left[{ }^{8-16]}\right.}$ Covalent conjugation of a PEG chain to siRNA has shown to increase serum stability of siRNA with sufficient gene silencing efficiency. ${ }^{[10,11]}$ Similar to the most of the protein PEGylation studies, all PEGylation strategies for siRNA delivery have been limited to the use of linear $P E G,{ }^{[8-15]}$ and in one case, six-arm PEG. ${ }^{[16]}$

Protein PEGylation studies have revealed that branched PEG can be more effective in enhancing the biological half- 
life of protein therapeutics by providing enhanced resistance against proteolysis and reduced immunogenicity due to its umbrella-like shape. ${ }^{[6,17,18]}$ Recently, comb-like PEG polymers, i.e., polyethylene glycol grafted poly(meth)acrylates, have proved to be potent alternatives to linear and branched PEG for improving the pharmacokinetics of protein drugs. ${ }^{[18-20]}$ When compared to the linear PEG with equivalent molecular weights, the comb-like PEG displays greater steric hindrance, thus potentially enhancing biostability and lowering clearance rates of the conjugated biotherapeutics. Moreover, comb-shaped PEG(meth)acrylate polymers can be prepared by reversible addition fragmentation chain transfer (RAFT) polymerization $^{[21-25]}$ and atom transfer radical polymerization (ATRP) techniques, ${ }^{[26-29]}$ which offer facile synthetic routes to the generation of polymers with controlled molecular weight, varying macromolecular architectures and defined endgroup functionalities. While control over the molecular weight and macromolecular structure of the conjugated polymer is crucial for improved pharmacokinetic properties of the bioconjugates, the defined end-group functionality enables varying synthetic strategies to be implemented for site-specific PEGylation.

Recently, we demonstrated the straightforward synthesis of reversible, well-defined siRNA-poly(polyethylene glycol methyl ether acrylate) (p(PEGA)) conjugates via the RAFT polymerization technique. ${ }^{[30]}$ In the present study, we have investigated, for the first time, the in vitro serum stability and gene silencing activity of the siRNA-p(PEGA) conjugates. Briefly, a 5'-sense-thiol-modified siRNA targeting the enhanced green fluorescence protein (eGFP) gene was conjugated covalently with two different $p$ (PEGA) polymers having different molecular weights via a reversible disulphide linkage. The serum and nuclease stability, cytotoxicity and gene silencing activity of the low and high molecular weight conjugates (LMWC and HMWC, respectively) were investigated. The results are summarized below.

\section{Experimental Part}

Materials and methods are given in Supporting Information.

\section{Results and Discussion}

Pyridyldisulfide-terminated poly(PEG-acrylate) (p(PEGA)) synthesized by RAFT polymerization using a pyridyldisulfide-modified RAFT agent (Figure S1 and S2, Supporting
Information) was conjugated to a $5^{\prime}$-sense-thiol-modified siRNA ${ }^{[30]}$ that targets the gene expressing enhanced green fluorescence protein (eGFP) (Scheme 1). The conjugation of siRNA with the polymers (low molecular weight polymer $(\mathrm{LMWP})=6000 \mathrm{~g} \cdot \mathrm{mol}^{-1}$, polydispersity index or PDI $=1.10$ and high molecular weight polymer $(\mathrm{HMWP})=17400$ g. $\mathrm{mol}^{-1}, \mathrm{PDI}=1.19$ ) was evident in gel electrophoresis data by the shift of the bands (as a smear) to higher molecular weights compared to the free siRNA (Figure 1A). The higher molecular weight band in the unconjugated siRNA lane (Lane 1) was attributed to the disulphide-linked dimers of siRNA. The average conjugation yield calculated by measuring the intensity of the bands in Figure $1 \mathrm{~A}$ using Image software was $68 \pm 3 \%$ and $69 \pm 3 \%$ for low and high molecular weight conjugates (LMWC and HMWC), respectively.

The conjugation of $p$ (PEGA) to siRNA through a disulfide linkage should enable the release of siRNA from the polymer chain under reductive conditions such as those present within the cells. ${ }^{[31]}$ Both low and high molecular weight conjugates (LMWC and HMWC, respectively) were treated with glutathione at intracellular concentrations (10 mM) for $1 \mathrm{~h}$. The gel electrophoresis results (Figure 1B and C) showed that both conjugates were cleaved completely after treatment with glutathione.

The stability of the unmodified siRNA and its conjugates was tested in $80 \%$ active fetal bovine serum (FBS) (Figure S3-S6, Supporting information). The unmodified siRNA and thiol-modified siRNA were completely degraded within 10 and $48 \mathrm{~h}$, respectively (Figure 2A). The degradation of LMWC and HMWC was much slower. The band intensity of the conjugates did not show a profound change within $48 \mathrm{~h}$. At $72 \mathrm{~h}$, the HMWC degradation was only $16 \%$. A previous study $^{[32]}$ showed that the siRNA-linear PEG $\left(\bar{M}_{n} 5000\right)$ conjugates had higher stability in $50 \%$ fetal bovine serum compared to the naked siRNA; however, these conjugates completely degraded in $50 \%$ serum within $24 \mathrm{~h}$. The higher stability of the siRNA conjugates with PEG-based polymers can be attributed to the steric hindrance effect of the polymer preventing the interactions between siRNA and the nucleases. Although not directly comparable, the higher

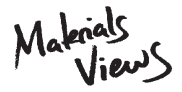

www.MaterialsViews.com 
A)

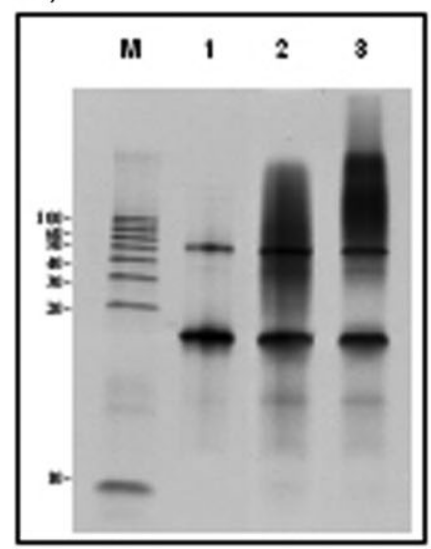

B)

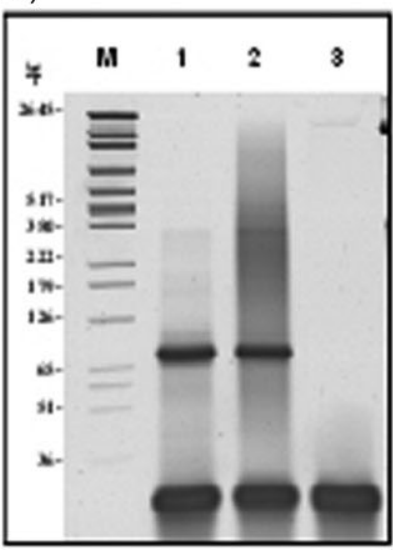

C)

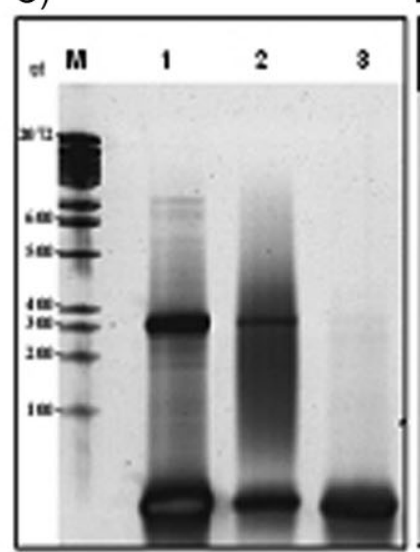

D)

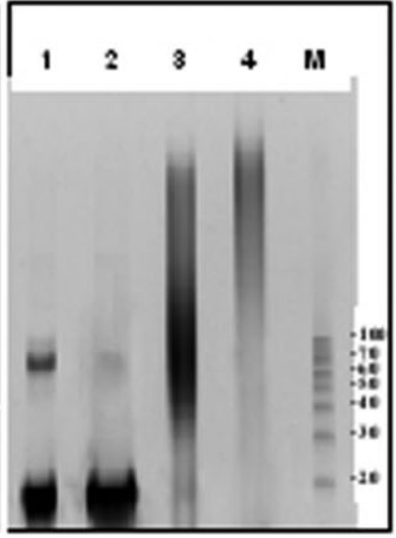

Figure 1. Polyacrylamide gel electrophoresis of (A) conjugation reaction mixtures, (B) and (C) siRNA-polymer conjugates (B-HMWC; C-LMWC) before and after reaction with $10 \mathrm{~mm}$ glutathione (GSH), (D) siRNA-polymer conjugates after HPLC purification and subsequent dialysis. (A) Lane 1 - unconjugated siRNA; Lanes 2 and 3 - LMWC and HMWC conjugation mixtures, respectively; M - 10 bp DNA marker. (B) Lane 1 unconjugated siRNA; Lanes 2 and 3 - HMWC before and after treatment with GSH, respectively; M - 36 bp DNA marker. (C) Lane 1 unconjugated siRNA; Lanes 2 and 3 - LMWC before and after treatment with GSH, respectively; M - Trackit 10o bp DNA marker. (D) Lane $1-$ unconjugated siRNA (containing disulfide crosslinked dimer); Lane 2 - unconjugated siRNA after treatment with $200 \mathrm{~mm}$ dithioerihtriol for $2 \mathrm{~h}$ (avoiding disulfide crosslinked dimer); Lanes 3 and 4 - purified LMWC and HMWC, respectively; Lane 5-10 bp DNA marker. All samples contained $0.03 \mathrm{nmol}$ siRNA.

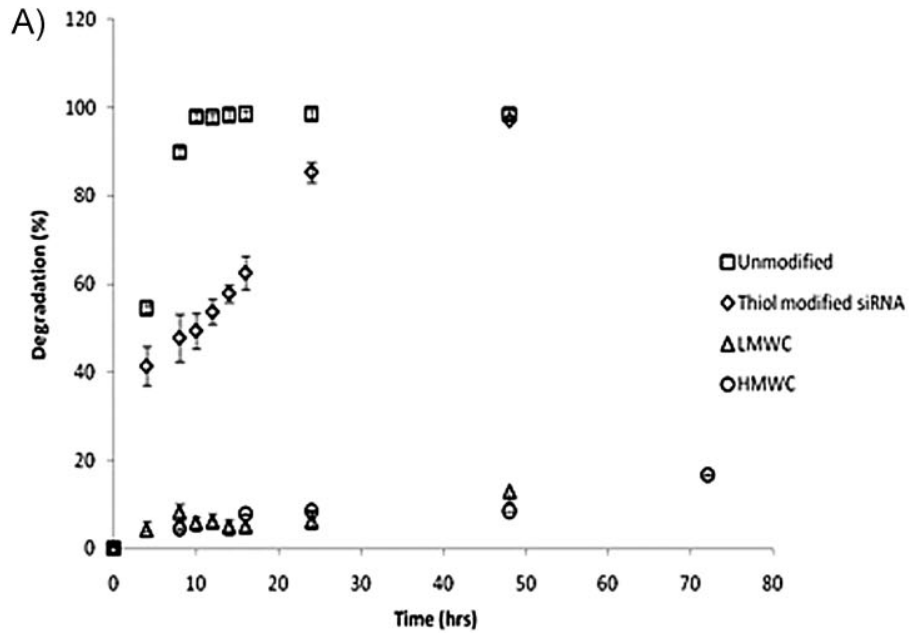

B)

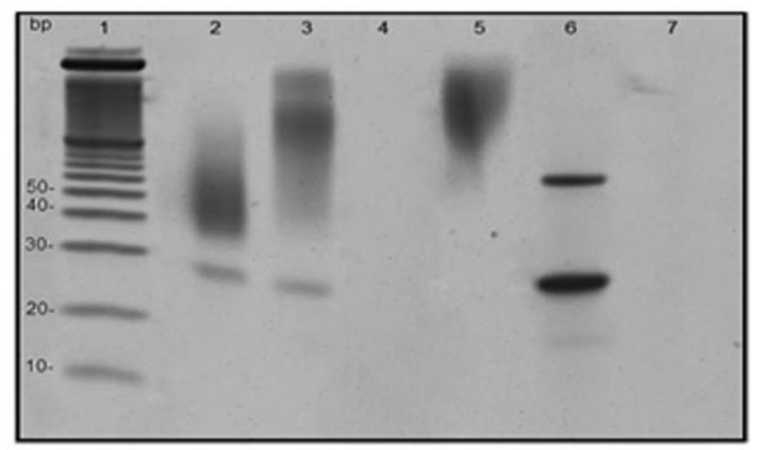

Figure 2. (A) Degradation of unmodified siRNA, thiol-modified siRNA, LMWC and HMWC in $80 \%$ active FBS, derived from PAGE data (Figure S3-S6, SI), as described in SI. The results are the average of three independent experiments \pm standard deviation. (B) Nuclease stability assay: Lane 1: Marker Lanes 2 and 4- LMWC before and after incubation with nuclease for 60 min, respectively; Lanes 3 and $5-$ HMWC before and after incubation with nuclease for $60 \mathrm{~min}$, respectively; Lanes 6 and 7 - unconjugated (thiol-modified) siRNA before and after incubation with nuclease for $60 \mathrm{~min}$, respectively.

serum stability of siRNA-p(PEGA) conjugates relative to siRNA-linear PEG conjugates can be attributed to the umbrella-like shape of $\mathrm{p}(\mathrm{PEGA})$, potentially providing greater protection to siRNA.

The stability of the conjugates was also tested against RNase $\mathrm{ONE}^{\mathrm{TM}}$ ribonuclease, a periplasmic enzyme that catalyzes the degradation of RNA by cleaving the phosphodiester bond between any two ribonucleotides. The unconjugated thiol-modified siRNA was completely degraded in $60 \mathrm{~min}$ (Figure 2B, Lane 6). The LMWC band mostly disappeared after 60 min (Lane 4). Interestingly, HMWC remained mostly stable within $60 \mathrm{~min}$. A control experiment that was performed to investigate the inhibitory effect of the $\mathrm{p}$ (PEGA), if there was any, on the ribonuclease activity showed that the unconjugated (thiol-modified) siRNA when simply mixed with the polymer (HMWP) (at a 1:1 molar ratio) resulted in complete degradation of the siRNA in $30 \mathrm{~min}$ after the incubation 
with the ribonuclease (Figure S7, ESI). The higher nuclease stability of the HMWC can be attributed to the greater steric hindrance of the higher molecular weight polymer chain relative to the lower molecular weight polymer.

Before proceeding to the cell culture experiments, semipreparative HPLC was performed to remove the unconjugated siRNA present in the conjugate mixtures. The PAGE analysis of the purified conjugates (Figure 1D) showed clearly that the free RNA band, previously present in the conjugate reaction mixtures in Figure $1 \mathrm{~A}$, was absent after the HPLC process.

The conjugates (LMWC and HMWC) were then formulated with a well-known fusogenic peptide KALA, ${ }^{[33]}$ (at an $(+/-)$ ratio of 2$)$ to investigate the gene silencing ability of the conjugates. KALA has proved to enable the endoplasmic escape of oligonucleotides. It has been previously demonstrated that KALA and oligonucleotides form complexes between 1.25 and $2.5(+/-)$ charge ratio and the $(+/-)$ ratio required to provide an efficient transfection is usually $10 .^{[33]}$ The formation of KALA complexes with siRNA-p(PEGA) conjugates was verified by PAGE (Figure S8, ESI).

Human neuroblastoma (SH-EP) cells were used as a model cell line in both cytotoxicity and gene silencing experiments. Considering the potential of polymer-based therapeutics in tumor treatments, SH-EP cell line was chosen as a suitable model for testing the polymer conjugates. A cell viability assay showed that lipofectamine alone caused the lowest cell viability (80\%). None of the other samples caused significant inhibition on the cell viability (>85\%) at the concentrations tested (Figure 3).

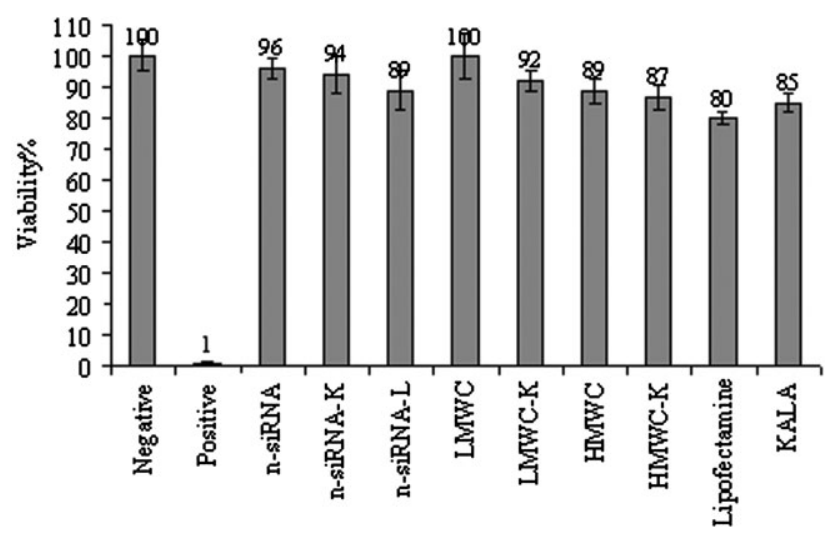

Figure 3. Viability of SH-EP cells incubated with unmodified siRNA ( $n$-siRNA), transfection agents (lipofectamine and KALA), n-siRNA complexes with KALA ( $n$-siRNA-K, $(+/-)=2$ ) or lipofectamine $(n-$ siRNA-L), the conjugates (LMWC and HMWC), LMWC or HMWC complex with KALA (LMWC-K, HMWC-K, respectively $(+/-)=2$ ) for $72 \mathrm{~h}$. The concentration of the samples was equivalent to $50 \mathrm{~nm}$ SiRNA. The negative and positive controls are the cells with no treatment and treated with Triton X-100 (2 wt.-\%), respectively. The results are the average of three independent experiments \pm standard error.
The protein expression inhibition of the conjugates was tested on SH-EP cells stably expressing eGFP (after $72 \mathrm{~h}$ posttransfection with the samples equivalent to $50 \mathrm{~nm}$ siRNA concentration). The fluorescence microscopy (Figure 4) showed that eGFP expression was not affected by the treatments with n-siRNA or n-siRNA-KALA complex. Contrary, siRNA-lipofectamine complex (siRNA-L) strongly reduced the fluorescence. Interestingly, the activity of the KALA complexes of both conjugates $((+/-)=2)$ on the eGFP expression was comparable with the activity of siRNA-L. The eGFP expression was also quantitatively investigated using flow cytometry (Figure 4, top graph). The KALA complex of the unmodified siRNA caused only $2 \pm 8 \%$ protein inhibition while the KALA complexes of LMWC and HMWC showed $56 \pm 5 \%$ and $58 \pm 3 \%$ eGFP inhibition, respectively. The protein inhibition caused by the KALA complexes of the LMWC and HMWC was comparable to the effect of the siRNA-lipofectamine complex (77 $\pm 7 \%)$. The flow cytometry results further revealed that the conjugates alone (both LMWC and HMWC) without a transfection agent inhibited the eGFP expression by $28 \pm 1 \%$ and $29 \pm 5 \%$, respectively, while the inhibition of the unmodified siRNA without a transfection agent was only $9 \pm 7 \%$. The combination of the protein inhibition and serum stability results suggest that the enhanced serum stability of the siRNA conjugated with $\mathrm{P}$ (PEGA)s might result in the enhanced protein inhibition.

\section{Conclusion}

In this study, we demonstrated, for the first time, the effects of the RAFT-synthesized comb-type PEG conjugation on siRNA's in vitro stability and gene inhibition activity. The results support the comb-like PEG (meth)acrylate polymers synthesized by RAFT polymerization as an effective component of delivery systems for siRNA. Since RAFT polymerization is versatile, one can envision preparing copolymers containing complexation and endosomal release functionality for siRNA delivery.

Acknowledgements: The authors acknowledge the Australian Research Council (ARC) for funding (DP 0770818). T.P.D. acknowledges ARC for Federation Fellowship. H.D.M. thanks the Sloan Foundation for additional funding. The authors thank Assoc. Prof M. Kavallaris (Children's Cancer Institute Australia for Medical Research) for providing SH-EP cells stably expressing eGFP.

Received: December 21, 2010; Revised: January 31, 2011; Published online: March 17, 2011; DOI: 10.1002/marc.201000804

Keywords: biological applications of polymers; PEG(meth)acrylate; polymer conjugates; reversible addition fragmentation chain transfer polymerization (RAFT); siRNA

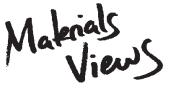

www.MaterialsViews.com
Macromol. Rapid Commun. 2011, 32, 654-659

(c) 2011 WILEY-VCH Verlag GmbH \& Co. KGaA, Weinheim 

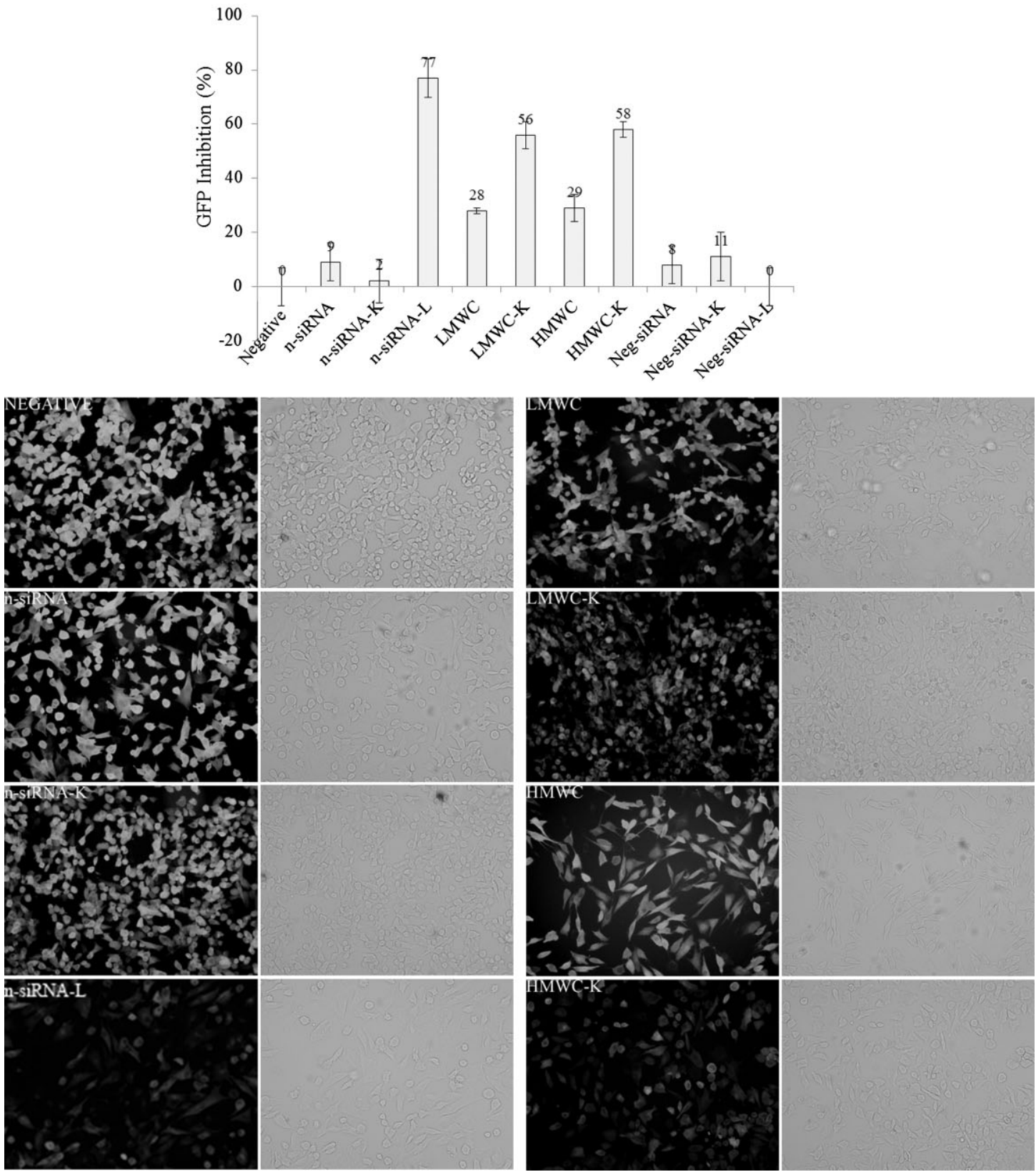

Figure 4. The eGFP inhibition (\%) results obtained by flow cytometry and representative epi-fluorescence micrographs of SH-EP cells at $72 \mathrm{~h}$ post-transfection without any treatment (negative), and with unmodified ( $n$-siRNA), its complexes with KALA $(n-s i R N A-K,(+l-)=2)$ and lipofectamine (n-siRNA-L), and with LMWC, LMWC complex with KALA (LMWC-K, $(+/-)=2)$, and HMWC and HMWC complex with KALA (HMWC-K, $(+/-)=2$ ). The concentration of the samples was equivalent to $50 \mathrm{nM}$ siRNA. In the upper graph, neg-RNA, and neg-siRNA-K and neg-siRNA-L are negative control siRNA and its complexes with KALA and lipofectamine, respectively. The results are the average of three different experiments \pm standard error.

[1] S. Crunkhorn, Nat. Rev. Drug Discov. 2010, 9, 359.

[2] K. A. Whitehead, R. Langer, D. G. Anderson, Nat. Rev. Drug Discov. 2009, 8, 129.

[3] Y.-K. Oh, T. G. Park, Adv. Drug Delivery Rev. 2009, 61, 850.

[4] L. Aagaard, J. J. Rossi, Adv. Drug Delivery Rev. 2007, 59, 75.

[5] D. B. Rozema, D. L. Lewis, D. H. Wakefield, S. C. Wong, J. J. Klein, P. L. Roesch, S. L. Bertin, T. W. Reppen, O. Chu, A. V. Blokhin, J. E. Hagstrom, J. A. Wolff, Proc. Natl. Acad. Sci. 2007, 104, 12982.
[6] S. M. Ryan, G. Mantovani, X. Wang, D. M. Haddleton, D. J. Brayden, Expert Opin. Drug Delivery 2008, 5, 371.

[7] J. M. Harris, N. E. Martin, M. Modi, Clin. Pharmacokinet. 2001, 40, 539

[8] M. Oishi, Y. Nagasaki, N. Nishiyama, K. Itaka, M. Takagi, A. Shimamoto, Y. Furuichi, K. Kataoka, ChemMedChem 2007, 2, 1290.

[9] S. H. Kim, J. H. Jeong, S. H. Lee, S. W. Kim, T. G. Park, J. Controlled Release 2008, 129, 107. 
[10] S. H. Kim, J. H. Jeong, S. H. Lee, S. W. Kim, T. G. Park, J. Controlled Release 2006, 116, 123.

[11] S. H. Lee, S. H. Kim, T. G. Park, Biochem. Biophys. Res. Commun. 2007, 357, 511.

[12] S. H. Kim, J. H. Jeong, S. H. Lee, S. W. Kim, T. G. Park, Bioconjug Chem. 2008, 19, 2156.

[13] M. Oishi, Y. Nagasaki, K. Itaka, N. Nishiyama, K. Kataoka, J. Am. Chem. Soc. 2005, 127, 1624.

[14] J. H. Jeong, H. Mok, Y.-K. Oh, T. G. Park, Bioconjug. Chem. 2008, 20, 5.

[15] T. Tatsumi, M. Oishi, K. Kataoka, Y. Nagasaki, S. Somiya, M. Doyama, Trans. Mater. Res. Soc. Japan 2008, 33, 807.

[16] S. W. Choi, S. H. Lee, H. Mok, T. G. Park, Biotechnol. Prog. 2010, 26, 57.

[17] C. Monfardini, O. Schiavon, P. Caliceti, M. Morpurgo, M. J. Harris, F. M. Veronese, Bioconjug. Chem. 1995, 6, 62.

[18] S. M. Ryan, X. Wang, G. Mantovani, C. T. Sayers, D. M. Haddleton, D. J. Brayden, J. Controlled Release 2009, 135, 51.

[19] W. Gao, W. Liu, J. A. Mackay, M. R. Zalutsky, E. J. Toone, A. Chilkoti, Proc. Natl. Acad. Sci. 2009, 106, 15231.

[20] C. T. Sayers, G. Mantovani, S. M. Ryan, R. K. Randev, O. Keiper, O. I. Leszczyszyn, C. Blindauer, D. J. Brayden, D. M. Haddleton, Soft Matter 2009, 5, 3038.
[21] G. Moad, E. Rizzardo, S. H. Thang, Aust. J. Chem. 2009, 62, 1402.

[22] C. Boyer, V. Bulmus, T. P. Davis, V. Ladmiral, J. Liu, Sb. Perrier Chem. Rev. 2009, 109, 5402.

[23] D. Pissuwan, C. Boyer, K. Gunasekaran, T. P. Davis, V. Bulmus, Biomacromolecules 2010, 11, 412.

[24] S. Kirkland-York, Y. Zhang, A. E. Smith, A. W. York, F. Huang, C. L. McCormick, Biomacromolecules 2010, 11, 1052.

[25] A. B. Lowe, C. L. McCormick, Prog. Polym. Sci. 2007, 32, 283.

[26] P. De, M. Li, S. R. Gondi, B. S. Sumerlin, J. Am. Chem. Soc. 2008, 130, 11288.

[27] K. Matyjaszewski, N. V. Tsarevsky, Nat. Chem. 2009, 1, 276.

[28] L. Tao, G. Mantovani, F. Lecolley, D. M. Haddleton, J. Am. Chem. Soc. 2004, 126, 13220.

[29] J. F. Lutz, H. G. Borner, K. Weichenhan, Macromolecules 2006 $39,6376$.

[30] K. L. Heredia, T. H. Nguyen, C.-W. Chang, V. Bulmus, T. P. Davis, H. D. Maynard, Chem. Commun. 2008, 28, 3245.

[31] R. E. Hansen, D. Roth, J. R. Winther, Proc. Natl. Acad. Sci. USA 2009, 106, 422.

[32] S. H. Kim, J. H. Jeong, S. H. Lee, S. W. Kim, T. G. Park, J. Controlled Release 2006, 116, 123.

[33] T. B. Wyman, F. Nicol, O. Zelphati, P. V. Scaria, C. Plank, F. C. Szoka, Biochemistry 1997, 36, 3008. 